\title{
Preliminary study in the analysis of the severity of cardiac pathologies using the higher-order spectra on the heart-beats signals
}

\author{
Sid Ahmed BERRAlH ${ }^{\mathrm{a}}$, Yettou Nour elhouda BAAKEK ${ }^{\mathrm{a}}$, Sidi Mohammed El Amine DeBBAL ${ }^{\mathrm{a},}$ \\ ${ }^{a}$ Biomedical Engineering Department, Faculty of Technology, Tlemcen University. Biomedical Engineering Laboratory \\ (GBM), Tlemcen, Algeria. BP 119 \\ *E-mail address: adebbal@yahoo.fr
}

\begin{abstract}
Phonocardiography is a technique for recording and interpreting the mechanical activity of the heart. The recordings generated by such a technique are called phonocardiograms (PCG). The PCG signals are acoustic waves revealing a wealth of clinical information about cardiac health. They enable doctors to better understand heart sounds when presented visually. Hence, multiple approaches have been proposed to analyze heart sounds based on PCG recordings. Due to the complexity and the high nonlinear nature of these signals, a computer-aided technique based on higher-order statistics (HOS) is employed, it is known to be an important tool since it takes into account the non-linearity of the PCG signals. This method also known as the bispectrum technique, can provide significant information to enhance the diagnosis for an accurate and objective interpretation of heart condition.

The objective expected by this paper is to test in a preliminary way the parameters which can make it possible to establish a discrimination between the various signals of different pathologies and to characterize the cardiac abnormalities.

This preliminary study will be done on a reduced sample (nine signals) before applying it subsequently to a larger sample. This work examines the effectiveness of using the bispectrum technique in the analysis of the pathological severity of different PCG signals. The presented approach showed that HOS technique has a good potential for pathological discrimination of various PCG signals.
\end{abstract}

Key words: phonocardiography; non-linearity; recordings; higher-order statistics; bispectrum.

\section{Introduction}

Cardiovascular diseases (CVDs) or heart disorders are the major cause of morbidity and mortality for men, women, and people of most ethnic and racial groups worldwide. Many people die each year from CVDs than from any other disease. According to the latest report from the World Health Organisation (WHO), deaths due to CVDs have reached almost 17.9 million worldwide. ${ }^{1}$ Early detection of CVDs is therefore of paramount importance and is carried out through medical devices for screening such as echocardiographs or perceiving the heart sounds by a cardiologist during auscultation. Auscultation is the action of listening to the noises that occur inside the body to make a diagnosis. However, the analysis of cardiac sounds by auscultation, based solely on the human ear, remains insufficient for a correct diagnosis of heart disease and for medical doctors to obtain all the information related to the mechanical activity of the heart. ${ }^{2}$

Nowadays, the vast technological explosion provides useful tools for understanding and recognizing normal and pathological heart sounds and murmurs. Considerable efforts have been devoted to exploring the sounds produced through cardiac structures by means of a simple technique, namely, phonocardiography. Phonocardiogram is a particularly useful diagnostic tool to detect and record cardiac sounds. PCG signal is a graphic record of acoustical waves that the heart produces during a cardiac cycle. The analysis of PCG signals using advanced computer-based signal processing techniques can provide clinicians with quantitative and qualitative information about the heart condition. This information can be the intensity of the heart sound, its location, frequency, quality and rhythm in the cardiac cycle.

In fact, the PCG signals are characterized by two principal acoustic events. The first heart sound (S1) is composed of several high components. It is the result of the closure of atrioventricular (mitral and tricuspid) valves. ${ }^{3}$ Like S1, the second heart sound (S2) is also composed of many components and its origin is attributed to the closure of the aortic and pulmonic valves. ${ }^{3}$ Apart from these two principal sounds, additional sounds may also be perceived such as murmurs. Heart murmurs are defined as sustained audible noises that are produced during the cardiac-beat cycle. ${ }^{4}$ 
In the most recent studies, several techniques and methods have been proposed to study the PCG signal in order to provide an aiding tool to help clinicians diagnose heart problems. Meziani et al. proposed a set of features using the Discrete Wavelet Transform (DWT) to discriminate between various heart sound signals. ${ }^{5}$

In this preliminary study, we focus on the analysis of the nonlinear characteristics of PCG signals through HOS method. Here, Bispectral-based features have been quantized that are important for quantifying changes between normal and pathological heart sounds. We will also provide various bispectral graphs for different cardiac sounds.

\section{Materials and Methods}

\section{Materials: Bispectral analysis}

Third-order spectral analysis or bispectrum is one of the robust methods applied for non-linear signal analysis. ${ }^{6}$ The Bispectral analysis is a modern signal processing technique that permits the extraction of non-linear characteristics and tracks the deviation of data from Gaussianity. ${ }^{7}$ The bispectrum $B\left(f_{1}, f_{2}\right)$ of a real process $\{x(k)\}$ represents the two-dimensional Fourier transform of the third-order correlation function of the signal and is given by Equation 1:

$B\left(f_{1}, f_{2}\right)=E\left[X\left(f_{1}\right) X\left(f_{2}\right) X^{*}\left(f_{1}+f_{2}\right)\right]$

Eq. 1

Where $B$ represents the bispectrum evaluated at the two frequencies $f_{1}$ and $f_{2}, X(f)$ is the Fourier transform of the signal $x(n K),{ }^{*}$ denotes the complex conjugation and $\mathrm{E}[$.] represents the statistical expectation operator. For deterministic sampled signals, $\mathrm{X}(\mathrm{f})$ is the discrete-time Fourier transform and is computed as the discrete Fourier transform (DFT) using the FFT algorithm. Frequencies can be normalized to be between 0 and 1 using the Nyquist frequency.

Note that the bispectrum $B\left(f_{1}, f_{2}\right)$ is a function of two frequencies, and therefore it can detect the phase coupling between two frequencies. ${ }^{8}$ Unlike the power spectrum, which suppress the phase information and can only describe linear mechanisms, bispectrum exclusively measures the correlation of phases between the frequency components $f_{1}, f_{2}$ and $\left(f_{1}+\right.$ $f_{2}$ ). The bispectrum is calculated in the triangular area $\Omega$, Namely the non-redundant region (see Figure 1), This nonredundant region is defined with the triangle $0 \leq f_{2} \leq f_{1} \leq f_{1}+$ $f_{2} \leq 1$.

\section{Experiment}

\section{HOS-Features extraction}

Higher-order spectra are the spectral representations of higherorder moments or cumulants of a signal. ${ }^{11}$ The third-order spectrum, also called bispectrum can be used for the analysis of PCG signals. In order to discriminate the distribution of bispectrum plots of these signals, a set of quantitative features must be defined. In this paper, several bispectrum-based features were calculated from the bispectral density array based on the works ${ }^{13,14}$ from the non-redundant region $\Omega$ (illustrated in Figure 1), also known as the principal domain.

Linear features corresponding to the amplitude changes have been used commonly to discriminate between similar spectra. For this purpose, the mean $\left(\mathrm{M}_{\mathrm{amp}}\right)$, amplitude variability $\left(\mathrm{A}_{\mathrm{var}}\right)$, maximum (Max), and minimum (Min) of the bispectrum magnitude have been proposed recently ${ }^{9,13,14}$ and described below.

1. The mean-magnitude of the bispectrum is defined by Equation 2:

$M_{a m p}=\frac{1}{L} \sum_{\Omega}\left|B\left(f_{1}, f_{2}\right)\right|$ Eq. 2

2. Bispectrum-magnitude variability is given by Equation 3:

$A_{\text {var }}=\frac{1}{L} \sum_{\Omega}\left(\left|B\left(f_{1}, f_{2}\right)\right|-M_{\text {ave }}\right)$

3. The maximum and the minimum of the bispectrum magnitude are defined by Equation 4 and Equation 5:

$\operatorname{Max}=\max \sum_{\Omega}\left(B\left|\left(f_{1}, f_{2}\right)\right|\right.$

Eq. 4

Min $=\min \sum_{\Omega}\left|B\left(f_{1}, f_{2}\right)\right|$

Eq. 5

In an attempt to measure the regularity or irregularity of PCG signals from bispectral plots, we proposed bispectral entropies. They have been previously used for automatic discrimination of biomedical signals, ${ }^{9,13}$ and were employed in this work.

4. Bispectral entropies BE1, BE2, and BE3 are calculated and expressed by Equations 6- 8 as follows:

$B E 1=-\sum_{n} p_{n} \log p_{n}$ Eq. 6 where $p_{n}=\frac{\left|B\left(f_{1}, f_{2}\right)\right|}{\sum_{\Omega}\left|B\left(f_{1}, f_{2}\right)\right|} \Omega$ : the non-redundant region in Fig. 1.

$B E 2=-\sum_{n} q_{n} \log q_{n}$ Eq. 7 where $q_{n}=\frac{\left|B\left(f_{1}, f_{2}\right)\right|^{2}}{\sum_{\Omega}\left|B\left(f_{1}, f_{2}\right)\right|^{2}} \Omega:$ the non-redundant region in Fig. 1. $B E 3=-\sum_{n} r_{n} \log r_{n}$ Eq. 8 where $r_{n}=\frac{\left|B\left(f_{1}, f_{2}\right)\right|^{3}}{\sum_{\Omega}\left|B\left(f_{1}, f_{2}\right)\right|^{3}} \Omega$ : the non-redundant region in Fig. 1.

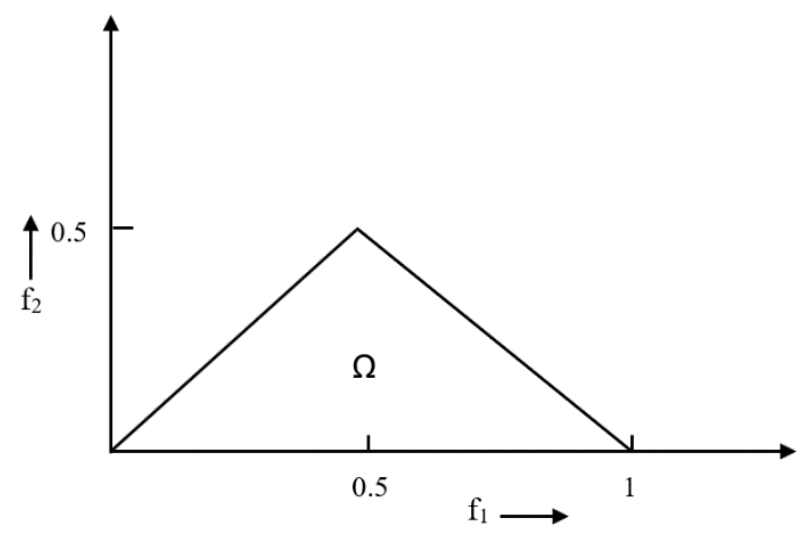

Figure 1. Non-redundant region $(\Omega)$ for computation of the bispectrum 
The normalization in Equations 6-8 above shows that entropies were computed for a parameter that lies between 0 and 1 (as required of probability).

Furthermore, bispectral phase entropy is also quantized in order to measure the degree of data disorder, and computed inside the bispectrum area $\Omega$.

5. The phase entropy is given by Equation 9: ${ }^{15}$

$P_{e}=\sum_{n} p\left(\Psi_{n}\right) \log p\left(\Psi_{n}\right)$

where $\Psi_{n}=\{\Phi \mid-\pi+2 \pi n / N \leq \Phi<-\pi+2 \pi(\mathrm{n}+1) / N\}$, $\mathrm{n}=0,1, \ldots, \mathrm{N}-1$,

$p\left(\Psi_{n}\right)=\frac{1}{L} \sum_{\Omega} 1\left(\phi\left(b\left(f_{1}, f_{2}\right)\right) \in \Psi_{n}\right)$

where $\Omega$ is the region as shown in Figure 1, $L$ corresponds to the total number of points within the non-redundant region, $\Phi$ is the phase angle of the bispectrum and 1(.) refers to the function which obtains a value of 1 when $\Phi$ is within the range bin $\Psi_{n}$ depicted in Equation 10. ${ }^{16}$

In our work, we have also employed the Weighted Center of Bispectrum (WCOB) in order to characterize the moment of the plot. ${ }^{17,18}$ From the non-redundant region, the WCOB are derived and shown as follow:

6. the sum of logarithmic amplitudes of the bispectrum:

$H_{1}=\sum_{\Omega} \log \left(B\left|\left(f_{1}, f_{2}\right)\right|_{1}\right)$

7. the sum of logarithmic amplitudes of diagonal elements in the bispectrum:

$H_{2}=\sum_{\Omega} \log \left(B\left|\left(f_{k}, f_{k}\right)\right|\right)$

Eq. 12

8. the first-order spectral moment of amplitudes of diagonal elements in the bispectrum:

$H_{3}=\sum_{k=1}^{N} k \log \left(B\left|\left(f_{k}, f_{k}\right)\right|\right)$

Yogesh CK et al. employed these features $(\mathrm{H} 1-\mathrm{H} 3)$ to recognize emotion and stress from a speech signal. ${ }^{18}$

9. The following equations are used to calculate the WCOB and the absolute values of $\mathrm{WCOB}^{18}$ (see Equation 14 and Equation 15):

$f_{1 m}=\frac{\sum_{\Omega} i B(i, j)}{\sum \Omega B(i, j)} f_{3 m}=\frac{\sum_{\Omega} i|B(i, j)|}{\sum \Omega|B(i, j)|}$

Eq. 14

$f_{2 m}=\frac{\sum_{\Omega} j B(i, j)}{\sum_{\Omega} B(i, j)} f_{4 m}=\frac{\sum_{\Omega} j|B(i, j)|}{\sum_{\Omega}|B(i, j)|}$

$f_{1 m}=W_{\text {cobx }}$

$f_{2 m}=W_{\text {coby }}$

$f_{3 m}=a W_{c o b x}$

$f_{4 m}=a W_{\text {coby }}$

Note that $i, j$, denote the frequency bin index within the nonredundant region, $f_{1 m}, f_{2 m}$ represent the WCOB and $f_{3 m}, f_{4 m}$ represent the absolute values of WCOB.

\section{Energetic ratio}

To check the validity of the HOS-based parameters used in this work, the energetic ratio (ER) is selected as a reference parameter. It has been shown that ER is a reliable parameter for monitoring the pathological severity of the heart. ${ }^{19,20}$ High amplitude heart murmurs indicate a worrying situation such as those in aortic stenosis and drum rumble.

ER provides an idea of the relative energy of cardiac murmurs relative to other heart sounds $\mathrm{S} 1$ and $\mathrm{S} 2$ and is given by Equation 16:

$R E=\frac{E_{2}}{E_{1}+E_{2}} \times 100$ where $\left\{\begin{array}{c}E_{1}: \text { heart sounds energy } \\ E_{2}: \text { murmur energy }\end{array}\right\}$ Eq. 16

The values given by the above equation ensure that the ER is calculated as a percentage. A value of $100 \%$ corresponds to the complete dominance of murmurs over heart sounds.

In this paper, previously classified signals corresponding to normal and abnormal cardiac sounds have been used. The PCG recordings database was obtained from. ${ }^{22,23}$

\section{Results and Discussion}

A phonocardiogram is a common clinical test used to measure the mechanical activity of the cardiac valves during the systole and diastole of the heart cycle. PCG is one of the most important tests used by practicians to evaluate cardiac health with suspected or known cardiac disease. In order to help the diagnosis and management of cardiac disorders, several techniques such as STFT and DWT have been proposed and applied for characterizing PCG signals. This paper introduces an analysis of the different PCG signals based on bispectrum. Another good advantage, bispectrum preserve phase information of the signal, which is important for capturing the changes in the quadratic phase coupling among the different component of the signal.

In this preliminary study, the validation of the method is evaluated and proved using nine PCG signals organized on three distinct groups, the PCG signals of the three groups are enlisted in the Table 1.

Table 1. The values of the energetic ratio (RE) of the different phonocardiogram signals used

\begin{tabular}{ccc}
\hline \hline PCG signals & Abbreviation & RE (\%) \\
\hline PCG signals without murmurs & & \\
\hline Normal & $\mathrm{N}$ & \\
Innocent murmur & IM & \\
Coarctation of the aorta & CA & 1.548 \\
\hline$P C G$ signals with clicks & & 2.826 \\
\hline Ejection click & EC & 13.175 \\
Atrial gallop & AG & 18.365 \\
Opening snap & OS & 44.075 \\
\hline$P C G$ signals with murmurs & & 51.409 \\
\hline Aortic stenosis & AS \\
Drum rumble & DR & \\
Aortic Regurgitation & AR & \\
\hline \hline
\end{tabular}


1. The first group: PCG signals without murmurs showing a morphology similar to that of the normal PCG signal: Normal (N), Innocent Murmur (IM), and Coarctation of the Aorta (CA) as given in Figure 12.

2. The second group: PCG signals with short murmurs (click): Ejection Click (EC), Atrial Gallop (AG), and Opening Snap (OS) (see Figure 14).

3. The third group: PCG signals with additive murmurs: Aortic Stenosis (AS), Drum Rumble (DR), and Aortic Regurgitation (AR) (see Figure 16).

For the data acquisition, an electronic stethoscope is employed to record the PCG signals. The heart sounds recordings obtained were converted to digital signals by using a 16-bit A/D converter at a sampling frequency fs $=8012 \mathrm{~Hz}$.

The PCG signals are segmented into frames and divided into 256 points with an overlap of $50 \%$ (128-point segments). The bispectrum was generated based on de direct fast Fourier transform and its graph was obtained using the bispecd function available in the Matlab HOSA toolbox.21 Then the bispectrum-based features for each frame are extracted and averaged over all frames at the non-redundant region $\Omega$.

(a)

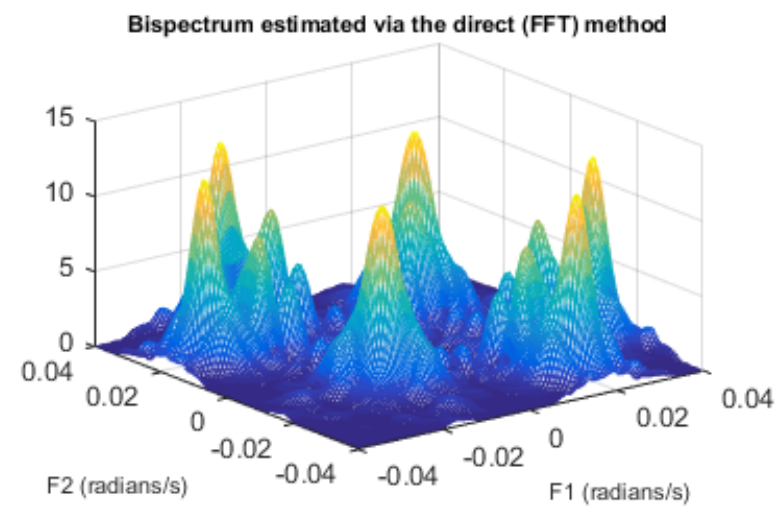

Figure 2. Typical plots for Normal (N) PCG: (a) Bispectrum, (b) Contour of (a)

\section{High order estimation using bispectrum}

Figures 2-10 show (a) the magnitude of the bispectrum and (b) the two-dimensional bispectral contour plot of typical normal (N), innocent murmur (IM), coarctation of the aorta (CA), Ejection click (EC), atrial galop (AG), opening snap (OS), aortic stenosis (AS), drum rumble (DR), and aortic regurgitation (AR) respectively. These plots are symmetric.

For the heart sounds signals of the first group (normal, innocent murmur, and coarctation of the aorta). The peaks in the bispectrum of the signal indicate the presence of phase coupling. Figure 2 (a) and (b) represent the bispectrum magnitude and its contour plot respectively for the normal heart sound signal. It can be seen from Figure 2(a) that the bispectrum of normal heart sound has a main lobe in the nonredundant region of significant magnitude. Furthermore, the bispectrum shows a magnitude distribution in the bi-frequency range from -0.04 to +0.04 . The magnitude of the bispectrum and its contour plot in the case of the innocent murmur (IM) are shown in Figures 3(a) and 3(b), respectively. It can be observed that the distribution of the bispectrum magnitude is different from the normal PCG.

(b)

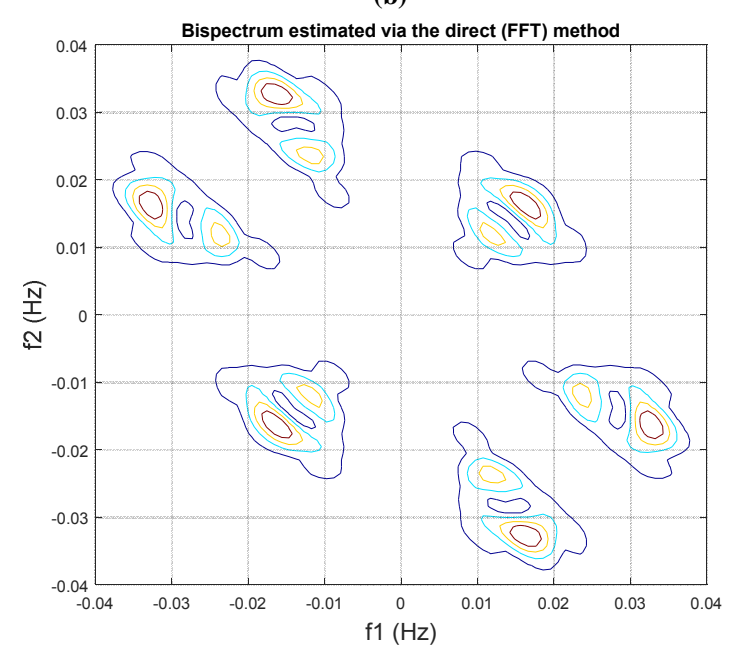

(b)

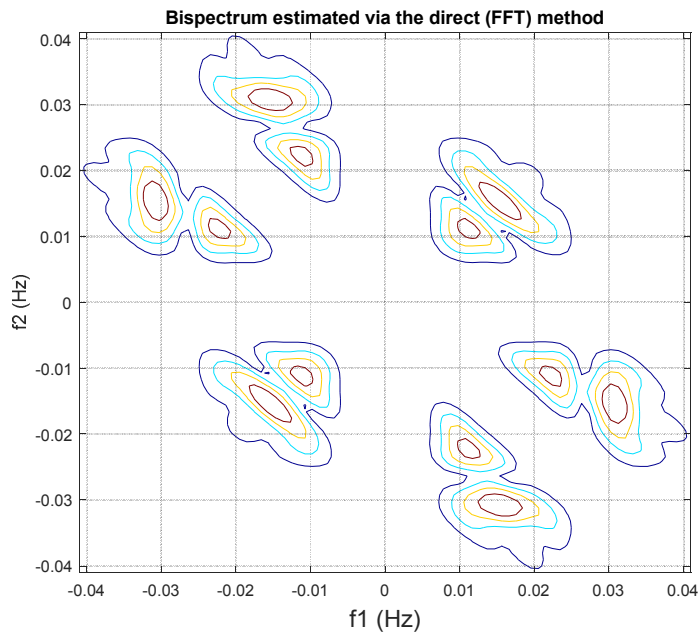

Figure 3. Typical plots for Innocent murmur (IM) PCG: (a) Bispectrum, (b) Contour of (a)

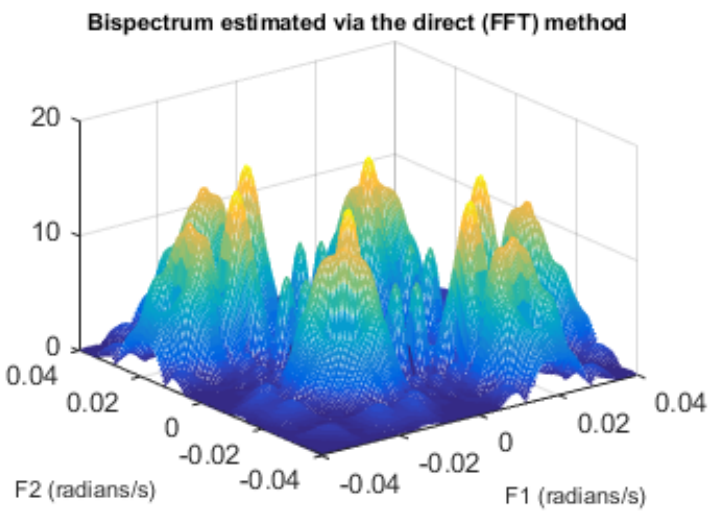


(a)

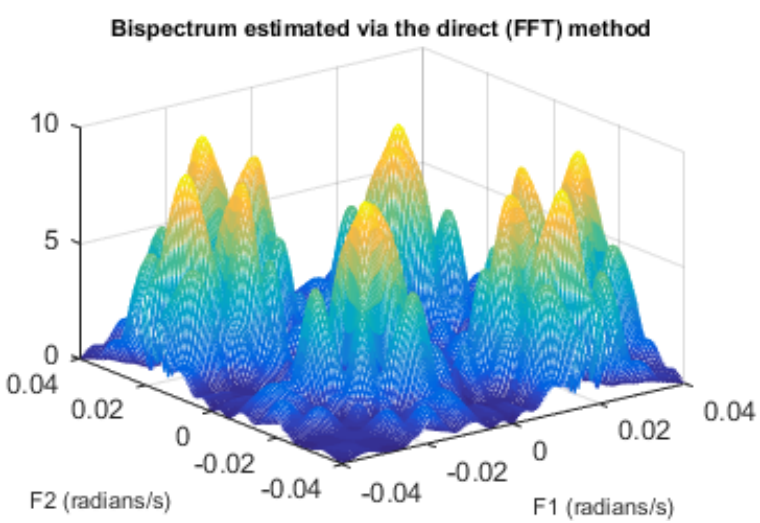

(b)

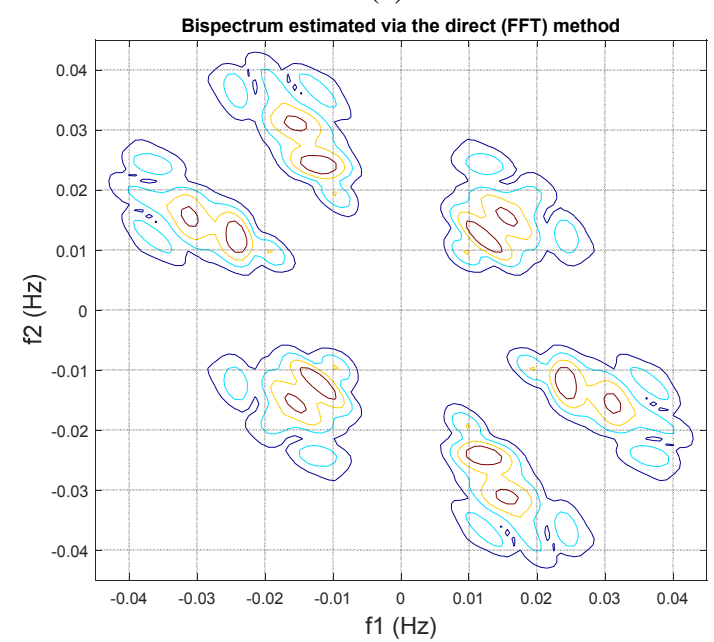

Figure 4. Typical plots for Coarctation of the aorta (CA) PCG: (a) Bispectrum, (b) Contour of (a)

(a)

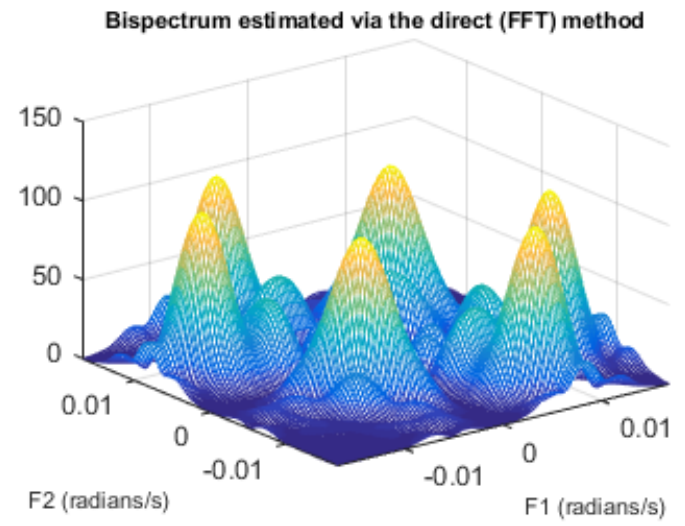

(b)

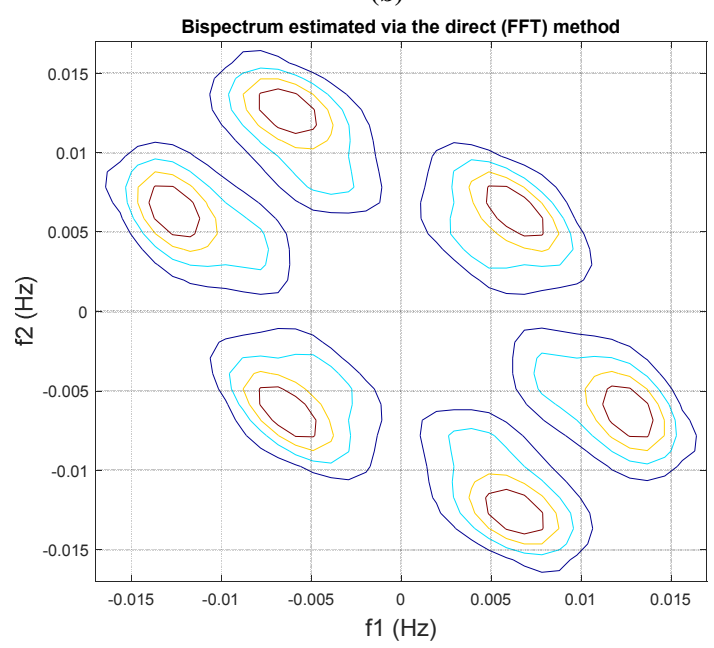

Figure 5. Typical plots for Ejection click (EC) PCG: (a) Bispectrum, (b) Contour of (a)

Similarly, Figures 4(a) and 4(b) show the magnitude of the bispectrum and its contour plot of the coarctation of the aorta (CA) signal. In Figure 4(a), the bispectrum plot presents a distribution of magnitude in the bifrequency range higher than then the normal and innocent murmur signals. Using the normal PCG as a benchmark, the range of the distribution of the bispectrum magnitude has gradually widened.

In addition, it can be noted that the bispectrum of MI and CA presents a morphological similarity to that of the normal case.

Figures 5-7 show the bispectrum analysis of PCG signals with short murmurs (EC, AG, OS). In particular, Figures 5 (a) and (b) depict the magnitude of the bispectrum and its contour map for the case of the ejection click, respectively. It has been noted that the distribution of the bispectrum on the plane $\left(f_{1}, f_{2}\right)$ shows a principal peak in the non-redundant region and other regions of symmetry. As can be seen, the bispectrum is distributed at phase-coupled frequencies between $-0.015 \leq f_{1}, f_{2}$ $\geq+0.015$. The bispectrum magnitude representation of atrial gallop (AG) and its contour plot are depicted in Figures 6(a) and 6(b). It can be observed that the bispectrum exhibits new peaks in the bifrequency range. In the case of opening snap (OS) PCG (see Figure 7(a) and 7(b)), it can be shown that bispectrum pattern is almost similar to that of ejection click signal. It can also be seen that the bispectrum magnitude of OS has peaks at high frequencies whereas no peaks were found on high frequencies for EC and AG signals. One noticed that the signals of this group show a spread of bispectrum magnitude in the bifrequency plane lower than that of the first group. The magnitude of the bispectrum is mainly between $-0.015 \leq f 1$, $f 2 \geq+0.015$ which is significantly lower than that of the normal PCG. This signifies that all three signals can be classified in the same group.

For the PCG signals of the third group AS, DR and AR heart sounds signals are selected. Figure 8(a) depicts the bispectrum magnitude plot and Figure 8(b) represents the corresponding bispectrum contour plot for aortic stenosis (AS) PCG. It can be noticed from the figure that the magnitude of the bispectrum plot provides peaks that are moved to lower frequencies. 
(a)

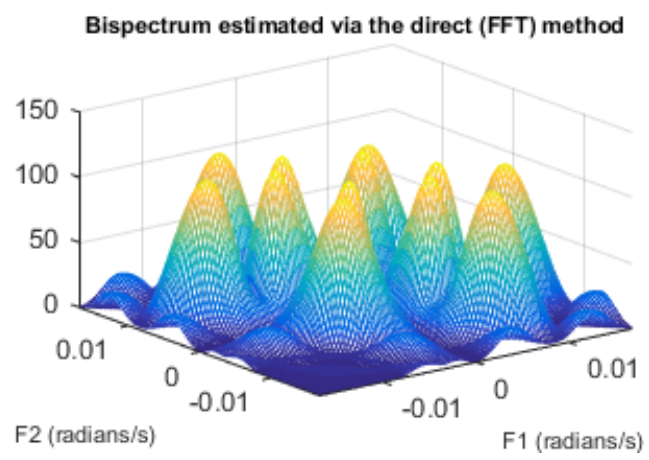

(b)

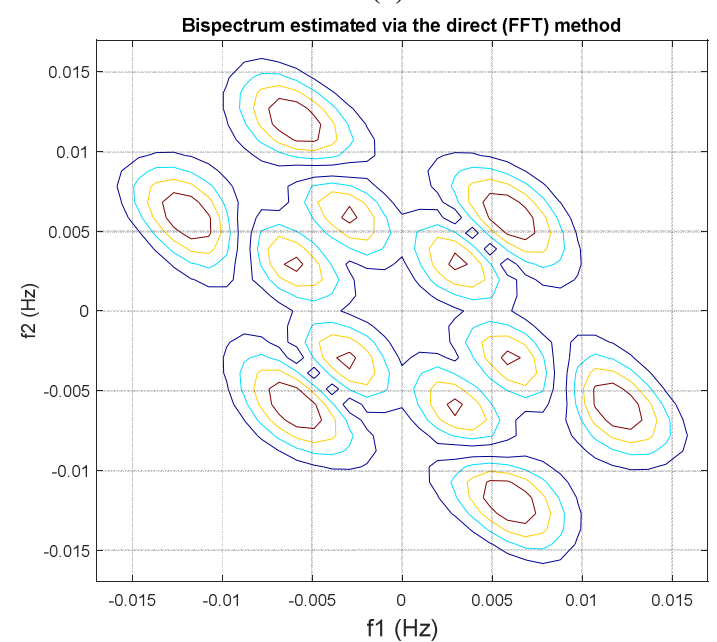

Figure 6. Typical plots for Arial gallop (AG) PCG: (a) Bispectrum, (b) Contour of (a)

(a)

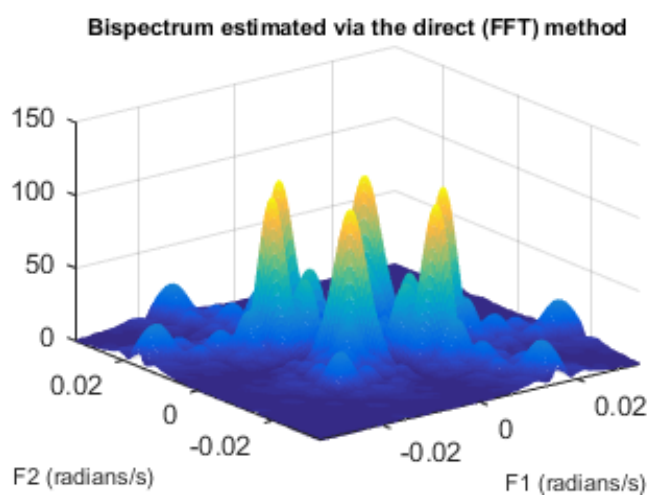

(b)

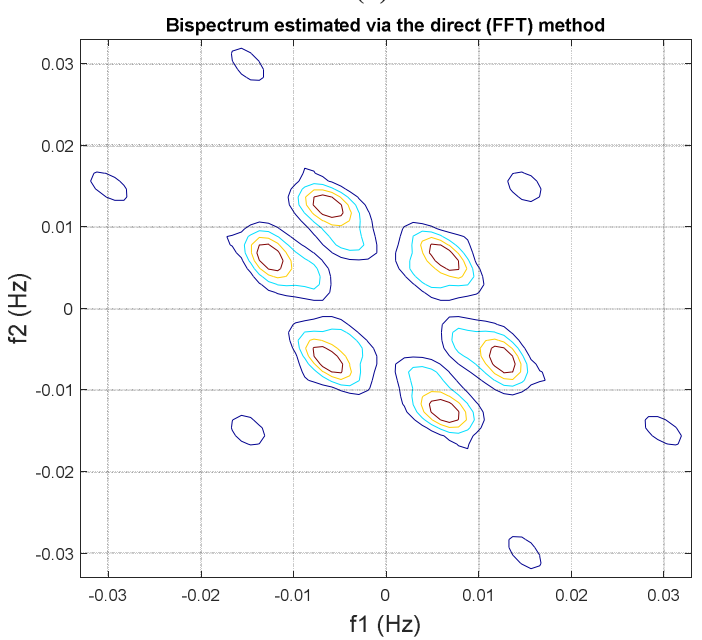

(b)

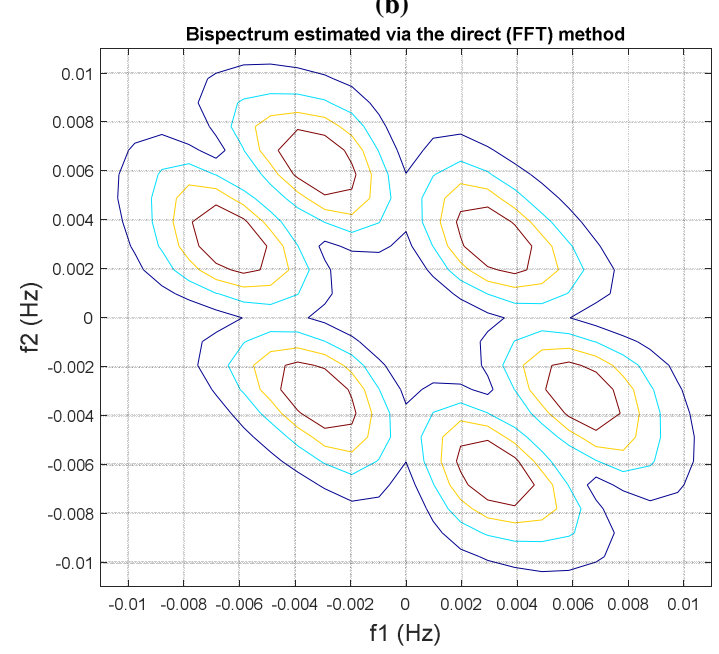

$$
\text { f1 }(\mathrm{Hz})
$$

Figure 8. Typical plots for Aortic stenosis (AS) PCG: (a) Bispectrum, (b) Contour of (a)

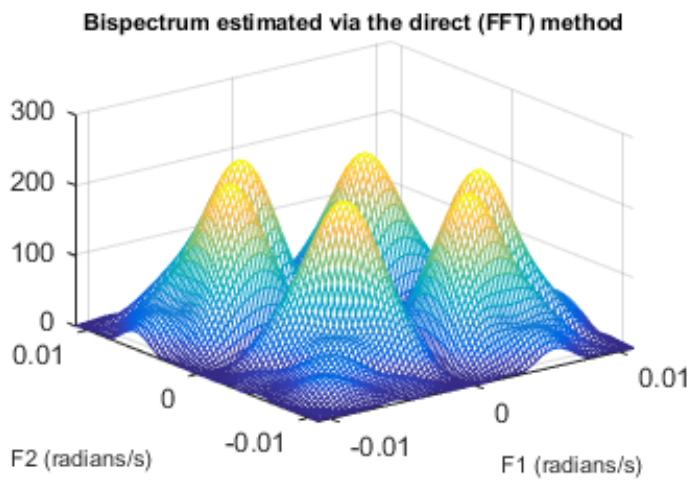


(a)

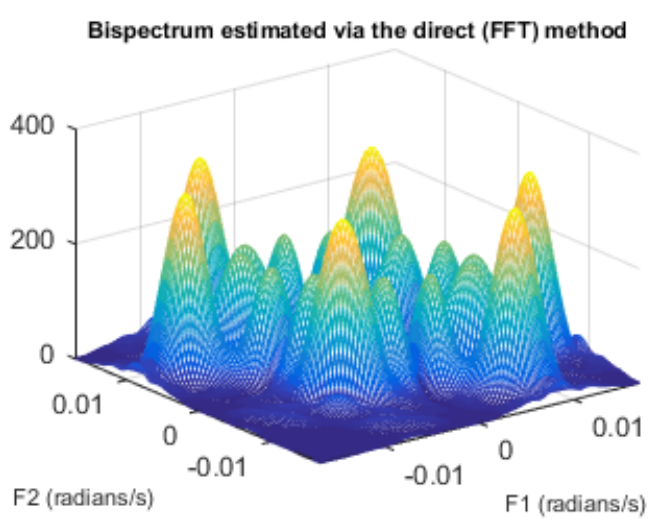

(b)

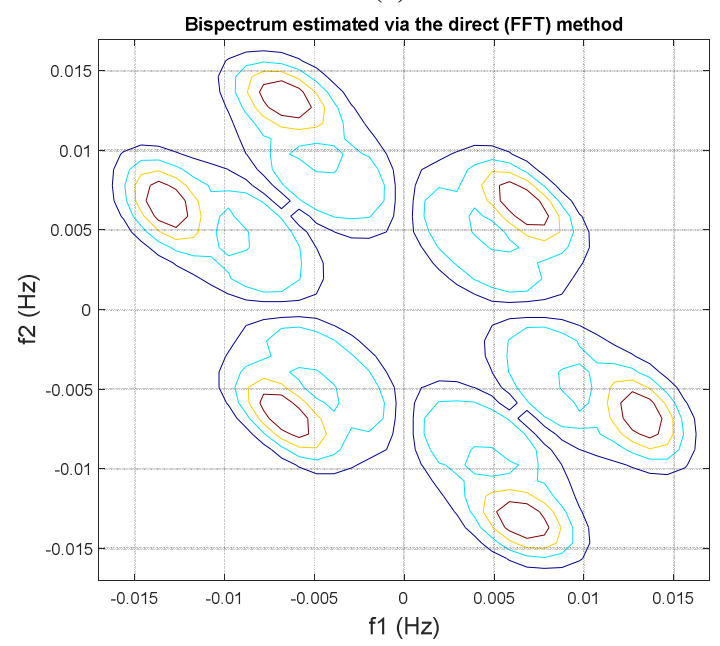

Figure 9. Typical plots for Drum rumble (DR) PCG: (a) Bispectrum, (b) Contour of (a)

(a)

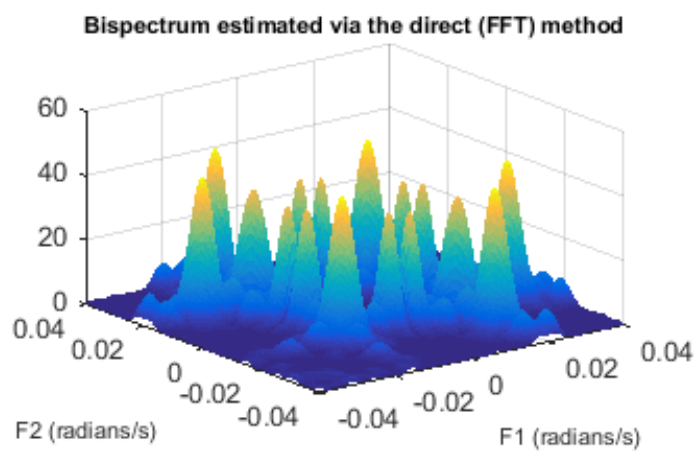

(b)

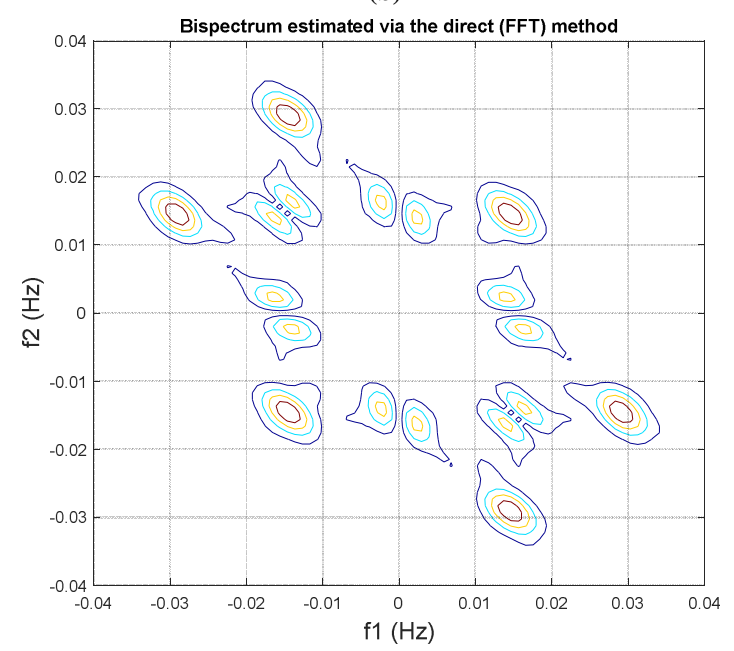

Figure 10. Typical plots for Aortic Regurgitation (AR) PCG: (a) Bispectrum, (b) Contour of (a)

When observing bispectral amplitude distribution, it can be seen that the bispectrum peaks are in the bifrequency range between $-0.01 \leq f_{1}, f_{2} \geq+0.01$. Figures 9(a) and 9(b) show the bispectral magnitude of the drum rumble (DR) signal and its contour plot representation. It can be observed that bispectral peaks appear within the bifrequency plane of $-0.015 \leq f_{1}, f_{2} \geq$ +0.015 . Figure 10(a) and 10(b) illustrate the bispectrum magnitude and its corresponding contour plot for a patient with aortic regurgitation (AR). One can clearly observe that there is a larger magnitude distribution than in the cases of AS and DR, where the magnitude of the bispectrum plot has peaks at the bifrequency extent (range of frequencies) of $-0.035 \leq f_{1}, f_{2} \geq$ +0.035 . compared with the normal case, the PCG signals of the third group exhibit bispectral peaks in the low bifrequency range. Thus, these bispectrum-based plots were able to analyze the non-linearity of the PCG signals, and are therefore a useful tool for discriminating between the various heart sound signals. These bispectrum plots are unique presentations for a given cardiac sound recording.

\section{Higher-order parameters extraction}

The aim of this article is to study the phonocardiogram signals (PCG) presenting a different cardiac severity. To distinguish the difference between the PCG signals and thus estimate the evolution of their pathological severity, histograms of the variation of the above-mentioned parameters are given in Tables 2-4. In these tables, for each group of PCG signals, only those HOS-based parameters that gave a good correlation with the increase in the severity of the pathology were displayed.

For the HOS features obtained from the bispectrum, the results presented in Table 2 can be schematically summarized using the histograms in Figures 11 to better identify each PCG signal by well-defined amplitude. Figures 11(a-i) are respectively the histograms of the mean of the amplitude, the maximum, the phase entropy, the bispectral entropies (BE1, $\mathrm{BE} 2$, and BE3) and the absolut of the weighted center of the bispectrum (WCOBx and WCOBy) according to the different PCG signals of the first group (N, IM, and CA). 
According to the results found, for signals with a morphology similar to that of the normal PCG, it can be seen that the parameters mentioned as the mean of the bispectrum amplitude Mamp and the phase entropy Pe can be used. The bispectrumbased parameters variation clearly shows that the normal PCG recording $(\mathrm{N})$ has the lowest value which is completely normal meanwhile the highest value goes for the CA signal. Consequently, this allowed us to conclude that the innocent murmur case is less serious than the coarctation of the aorta. These calculated parameters clearly show the difference that can exist between the first group signals and hence qualify the bispectral analysis as the most reliable method of analysis to assess the cardiac pathological severity. This is in complete accordance with what has been found by using DWT method. ${ }^{5}$
Table 2. The HOS parameters values extracted from PCG signals of the second group (normal (N), innocent murmur (IM), and coarctation of the aorta $(\mathrm{CA}))$.

\begin{tabular}{cccc}
\hline \hline \multirow{2}{*}{ Features name } & \multicolumn{3}{c}{ PCG signals without murmurs } \\
\cline { 2 - 4 } & $\mathbf{N}$ & IM & CA \\
\hline Mamp & 0.005 & 0.014 & 0.028 \\
Avar & -0.143 & -0.414 & -0.843 \\
Max & 0.024 & 0.126 & 0.616 \\
BE1 & 0.695 & 0.519 & 0.395 \\
BE2 & 0.395 & 0.341 & 0.229 \\
BE3 & 0.307 & 0.276 & 0.139 \\
Pe & 1.206 & 2.148 & 2.421 \\
absolut Wcobx & 18.821 & 10.474 & 6.493 \\
absolut Wcoby & 6.254 & 5.155 & 4.245 \\
\hline \hline
\end{tabular}

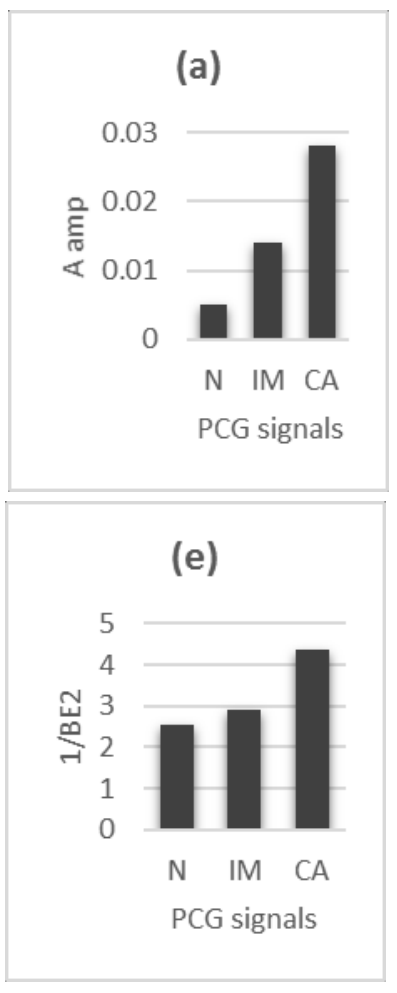

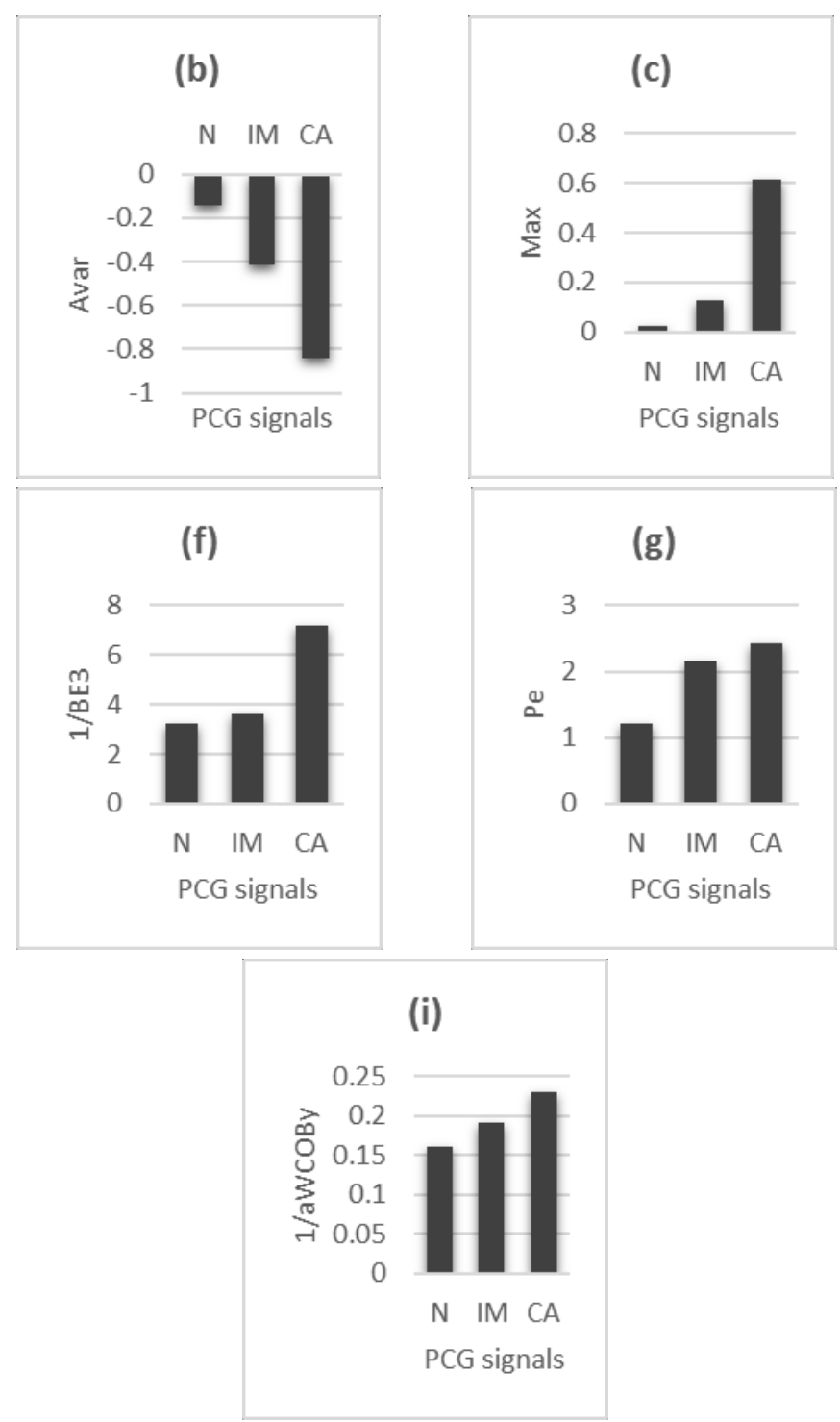

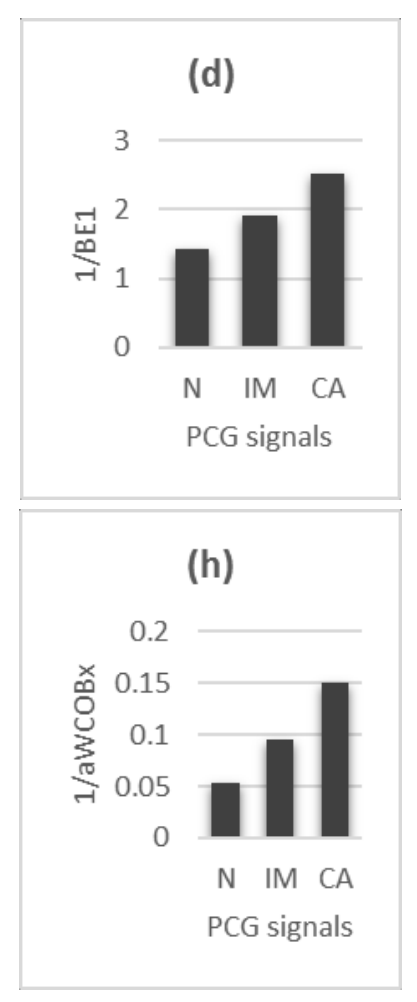

Figure 11. (a-i) - bispectral parameters variation extracted from PCG signals of the first group (normal (N), innocent murmur (IM), and coarctation of the aorta (CA)), ((a) the mean of the amplitude Mamp, (b) bispectrum variability Avar, (c) maximum of the bispectrum Max, (d-f) inverse of normalized bispectral entropy 1/BE1,1/BE2, and 1/BE3, (g) phase entropy Pe, (h, i) inverse of weighted center 1/aWCOBx, 1/aWCOBy). 
(a)

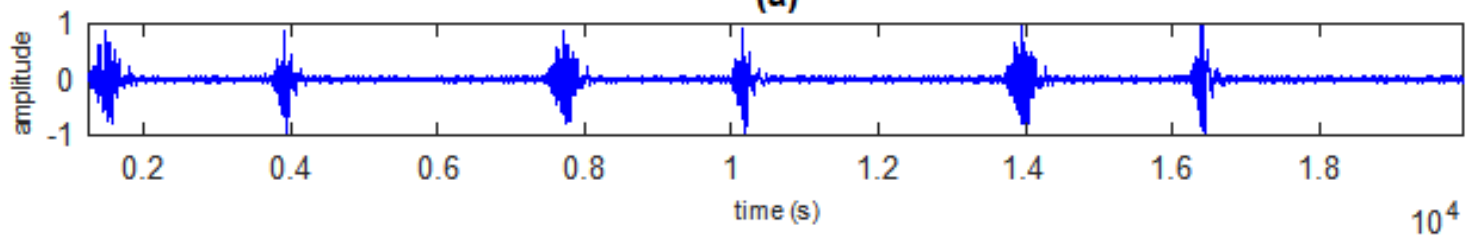

(b)

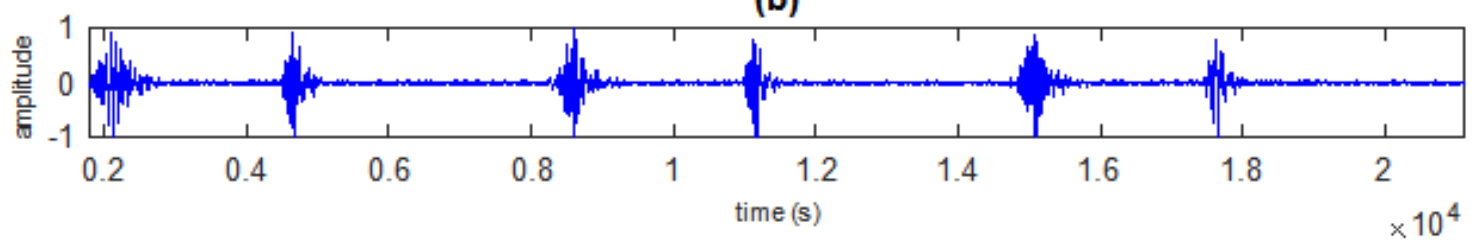

(c)

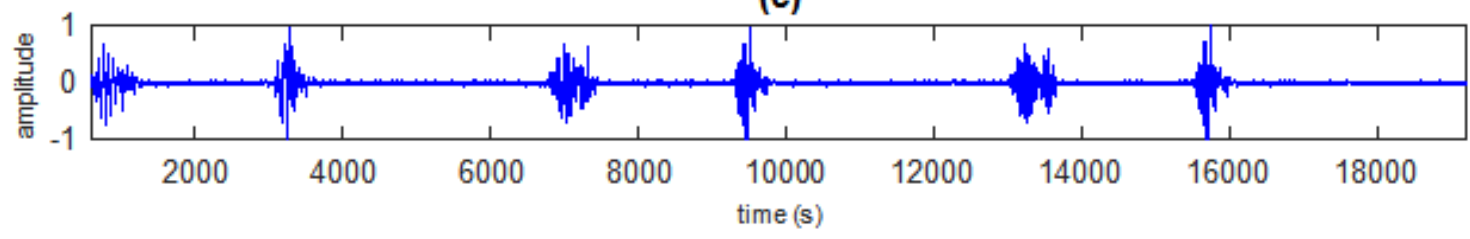

Figure 12. The PCG signal of the first group ((a) normal (N), (b) innocent murmur (IM), and (c) coarctation of the aorta (CA))

Concerning the selected PCG signals of the second group (EC, $\mathrm{AG}$, and $\mathrm{OS}$ ), the obtained HOS-based features have been illustrated in Table 3. Figures 13(a-g) give histogram plots of the amplitude variation of the bispectral entropies (BE1 and BE2), the phase entropy, the sum of logarithmic amplitudes, the first-order spectral moment of amplitudes of diagonal elements ( $\mathrm{H} 1$ and $\mathrm{H} 2)$, and the weighted center of the bispectrum (WCOBx and WCOBy), respectively as well as the graphs showing the variation of the energetic ration (ER). According to previous work, ${ }^{20,22}$ the ER parameter is known to be one of the most reliable parameters in the estimation of the murmur energy. From the graphs illustrating the variation of the energetic ratio in Figures 13 (a-g), it is noticed a progressive increase in the variation of the ER where the OS signal has the greater value of $13.18 \%$ followed by the AG signal $2.83 \%$ while the EC signal presents the lowest value $1.55 \%$ that allows considering this latter to be the less severe compared to the other cases.

As expected, it can be seen that the selected HOS parameters can serve to assess the pathological severity of the three PCG signals in this group as they show a good correlation with the variation in the amplitude of the energetic ratio. It is observed that these parameters evolve progressively from the ejection click (EC) signal to the opening snap (OS). Thus, it can be deduced that bispectrum parameters fully serve to differentiate between these three different signals (EC, AG, and OS).

For the analysis of the PCG signals presenting an important murmur (PCG of the third group AS, DR, and AR), the bispectrum algorithm was also computed and the corresponding parameters were extracted and displayed in Table 4. For this group, five parameters have been considered: the mean of the amplitude, the amplitude variability, the maximum, the bispectral entropy BE3, and the phase entropy. Figures 15 (a-e) illustrate the histograms that evolve according to the amplitude variation of the selected HOS parameters for this heart sounds signals group.

These figures also depict the variation of the energetic ratio which can be used as a reference parameter in the monitoring of cardiac pathology. It can be seen that the AS and DR signals (Figure 16) present lower values $18.37 \%$ and $44.07 \%$, respectively than that of AR signal which reaches $51.41 \%$. One can clearly notice that the HOS-based parameters chosen evolve progressively from the aortic stenosis (AS) signal to the aortic regurgitation (AR). Consequently, the HOS-based parameters can faithfully give a clear view of pathological severity evolution as they show a relative proportionality with the variation of the energetic ratio (ER).

Table 3. The HOS parameters values extracted from PCG signals of the second group (ejection click (EC), atrial gallop (AG), and opening snap (OS)).

\begin{tabular}{cccc}
\hline \hline \multirow{2}{*}{ Features name } & \multicolumn{3}{c}{ PCG signals with clicks } \\
\cline { 2 - 4 } & EC & AG & OS \\
\hline BE1 & 0.7196 & 0.7967 & 0.7972 \\
BE2 & 0.0875 & 0.1079 & 0.4857 \\
Pe & 3.5786 & 3.5769 & 3.4528 \\
H2 & -337.0511 & -332.0326 & -254.0609 \\
H3 & -1011.1413 & -996.0846 & -762.1674 \\
Wcobx & 9.4133 & 20.2133 & 22.0821 \\
Wcoby & 1.4912 & 3.03655 & 7.1826 \\
\hline \hline
\end{tabular}




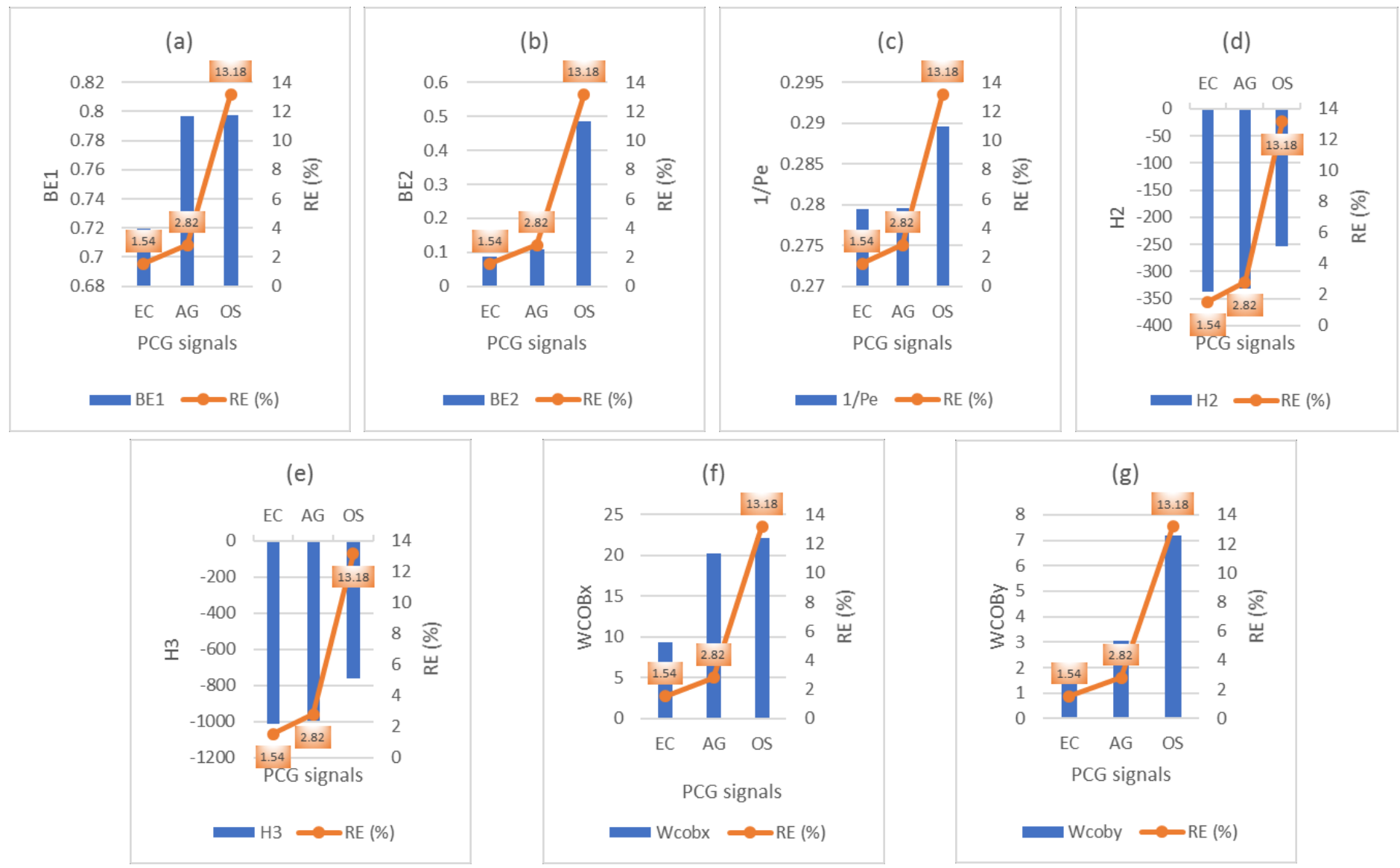

Figure13.(a-g) - bispectral parameters variation extracted from PCG signals of the second group (ejection click (EC), atrial gallop (AG), and opening snap (OS)), ((a) normalized bispectral entropy BE1, (b) normalized bispectral squared entropy BE2, (c) inverse of phase entropy 1/Pe, (d) sum log amplitude of diagonal element $\mathrm{H} 2$, (e) first order spectral moment of amplitude of diagonal element $\mathrm{H3}$, (f, g) weighted center WCOBx, WCOBy), and the energetic (ER) ratio variation

(a)

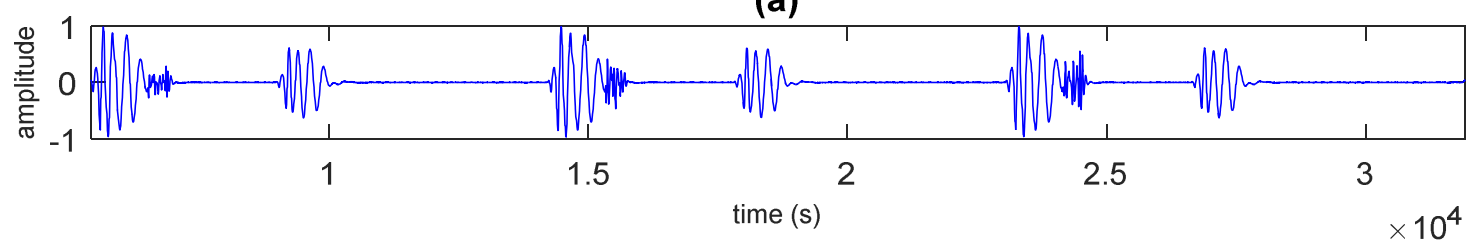

(b)

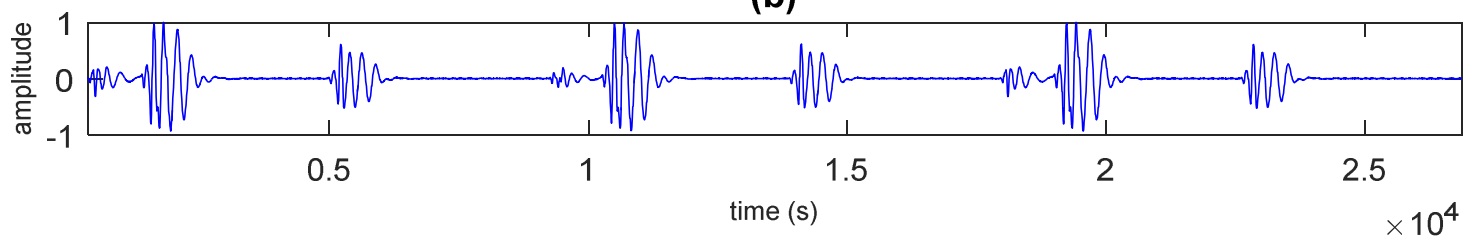

(c)

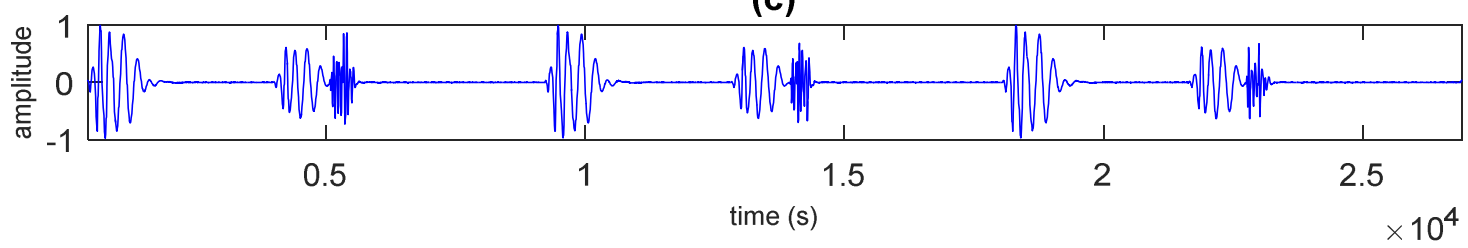

Figure14 - The PCG signals of the second group ((a) ejection click (EC), (b) atrial gallop (AG), and (c) opening snap (OS)), 

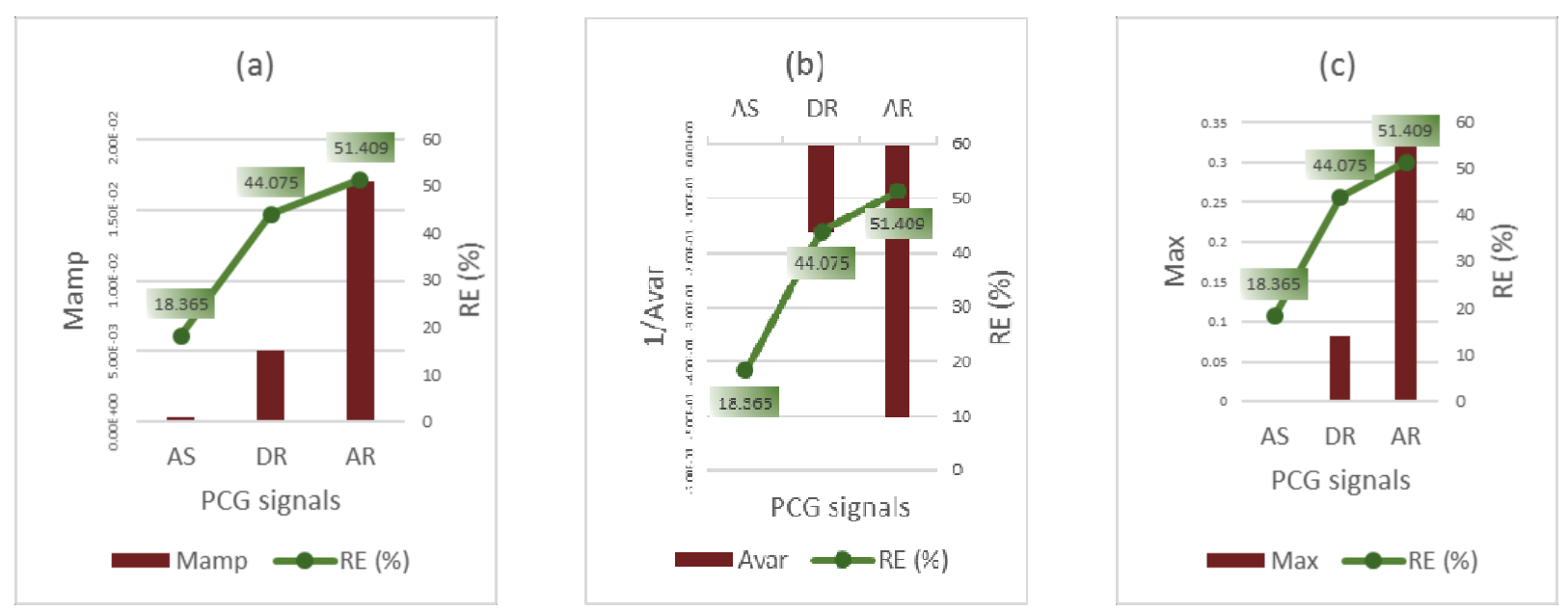

(d)

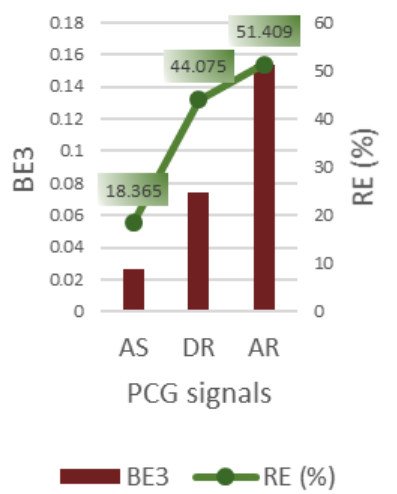

(e)

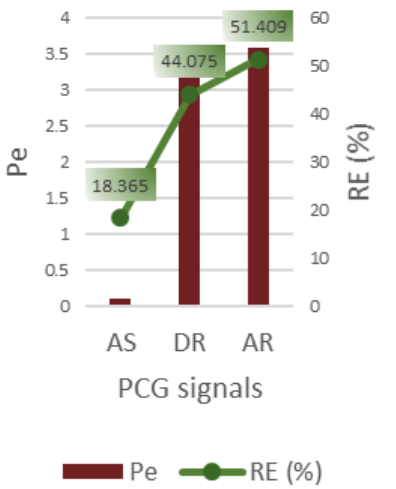

Figure15. (a-e) - bispectral parameters variation extracted from PCG signals of the third group (aortic stenosis (AS), drum rumble (DR), and aortic regurgitation (AR)), ((a) mean of the amplitude $M_{a m p}$, (b) inverse of bispectrum amplitude variability $1 / A_{v a r}$, (c) max of bispectrum Max, (d) normalized bispectral cubed entropy $\mathrm{BE}_{3}$, (e) phase entropy $\mathbf{P}_{\mathrm{e}}$ ), and the energetic (ER) ratio variation.

(a)

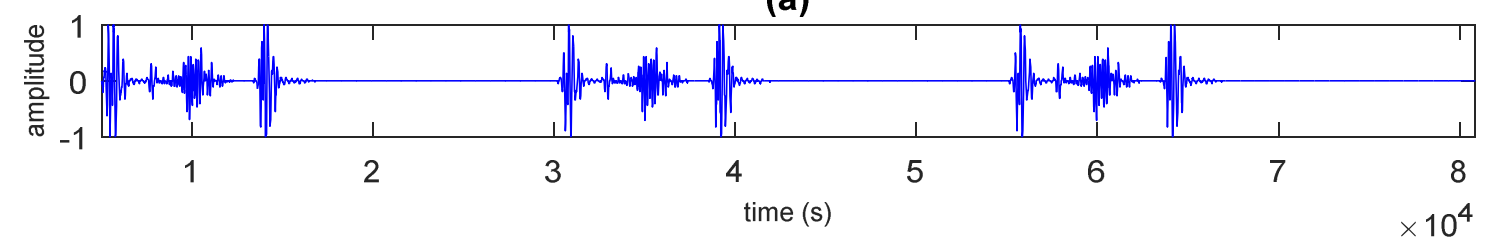

(b)

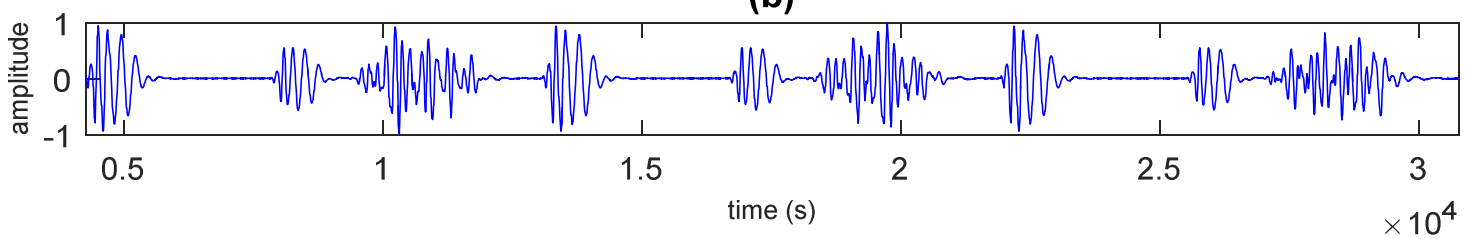

(c)

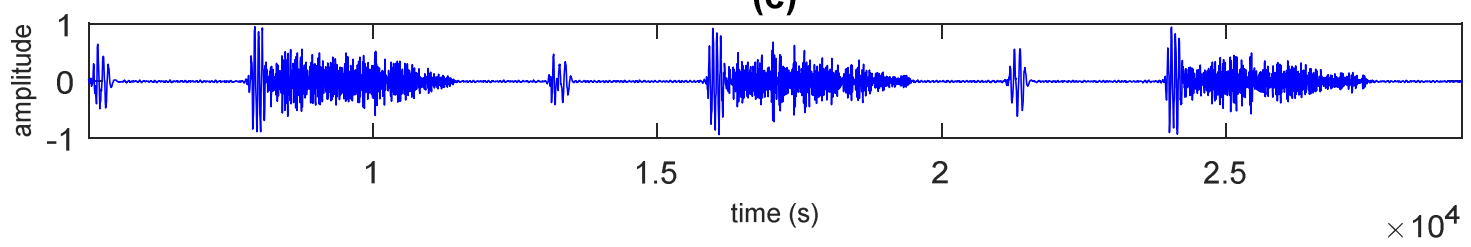

Figure 16 - The PCG signals of the third group ((a) aortic stenosis (AS), (b) drum rumble (DR), and (c) aortic regurgitation (AR)). 
Table 4. The HOS parameters values extracted from PCG signals of the third group (aortic stenosis (AS), drum rumble (DR), and aortic regurgitation (AR)).

\begin{tabular}{cccc}
\hline \hline \multirow{2}{*}{ Features name } & \multicolumn{3}{c}{ PCG signals with murmurs } \\
\cline { 2 - 4 } & AS & DR & AR \\
\hline Mamp & $3.298 \mathrm{E}-04$ & 0.005 & 0.017 \\
Avar & $-5.249 \mathrm{E}-04$ & -0.163 & -0.502 \\
Max & 0.001 & 0.082 & 0.319 \\
BE3 & 0.026 & 0.074 & 0.154 \\
Pe & 0.107 & 3.375 & 3.579 \\
\hline \hline
\end{tabular}

Table 5. The HOS parameters and the energetic ratio parameter values extracted from the mitral prolapse PCG (MP).

\begin{tabular}{ccccc}
\hline \hline & \multicolumn{4}{c}{ PCG signals } \\
\hline Features name & MP1 & MP2 & MP3 & MP4 \\
\hline ER & 14,85 & 23,53 & 56,29 & 75,48 \\
H1 & 1.1115 & 1.2313 & 1.6000 & 3.7904 \\
H2 & 154.8896 & 176.4188 & 229.4135 & 578.5698 \\
\hline \hline
\end{tabular}

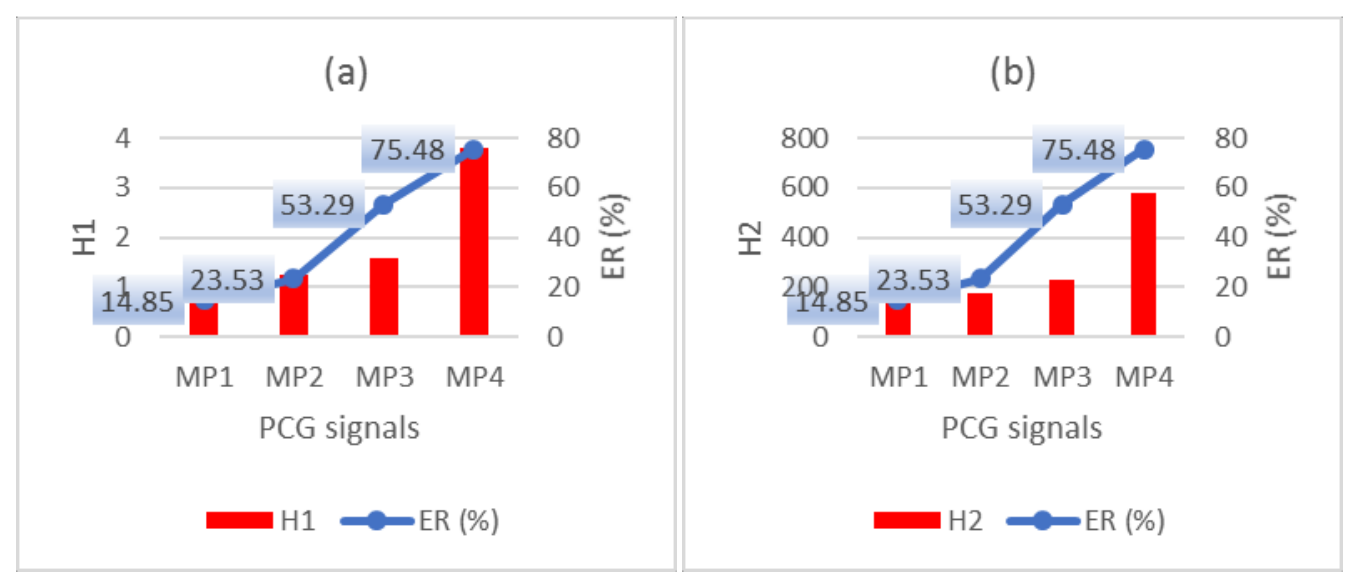

Figure 17 - The variation of the moment of the order (H1 and H2) and the energetic ratio extracted from the Mitral prolapse PCG (MP1, MP2, MP3, and MP4)

\section{Severity degree analysis of the pathological Phonocardiogram signals}

In this part of our article we will carry on an analysis of the severity degree of pathological phonocardiogram signals of a selected case with the calculation of certain parameters obtained from the application of the HOS technique (bispectrum) such as moments of order ( $\mathrm{H} 1$ and $\mathrm{H} 2)$.

The PCG signal chosen here in our application is Mitral Prolapse (MP) with four different and ascending severity degrees (MP1, MP2, MP3 and MP4). This growth was made possible by the use of the ER (Energetic Ratio) parameter.

These two parameters $\mathrm{H} 1$ and $\mathrm{H} 2$ have been calculated to see if they can follow the evolution and variation of the pathology (see Table 5). From the values obtained and given in Table 5, it is clear that the variation of the two chosen parameters $\mathrm{H} 1$ and $\mathrm{H} 2$ effectively follow the variation of the pathology (as does the ER parameter).

Under these conditions, these two parameters can be used not only to differentiate between different PCG signals but also to detect the degree of severity and its evolution in the same pathology.

To be effective, this must be verified against other PCG signals of different degree of pathological severity. This obviously requires a more thorough and targeted acquisition of these pathologies or a rich and varied database, sometimes difficult to find.

\section{Conclusion}

In this work, the bispectral technique has been carried out to analyse the cardiac sound signal. The bispectrum and its derived features were computed and employed for discrimination among the PCG cases. The bispectrum-graph representations were able to provide a unique pattern for each type of PCG signal. It is found that each of the PCG signals has a unique signature in bispectrum domain which is effectively used for the classification into different groups. We have also studied the different HOS features that can be used in the analysis of PCG signals. By using the energetic ratio as a standard parameter, the derived bispectrum parameters show a relative proportionality with the increasing evolution of the cardiac severity as they are able to increase in coherent way with the growing importance of the heart pathology.

We can therefore affirm that these preliminary results obtained from this study on a small number of signals but nevertheless of very different severity are very promising. A future application on a larger sample may refine the results and comfort the user in the use of this method to discriminate between various pathologies and ensure the importance of the severity involved. 


\section{Acknowledgments}

The authors would like to thank the Directorate-General of Scientific Research and Technological Development (Direction

Générale de la Recherche Scientifique et du Développement
Technologique, DGRSDT, URL:www.dgrsdt.dz, Algeria) for the financial assistance towards this research.

\section{References}

1. World Health Organization.Cardiovascular diseases. https://www.who.int/westernpacific/health-topics/cardiovascular-diseases.

2. Debbal SM. Computerized Heart Sounds Analysis. In: Discrete Wavelet Transforms: Biomedical Applications. IntechOpen, 2011. https://doi.org/10.5772/23700

3. Li X, Zhong L, Luo L, et al. Synchronization control of pulsatile ventricular assist devices by combination usage of different physiological signals. Comput Assist Surg. 2019;24:105-112. https://doi.org/10.1080/24699322.2018.1560089

4. Ahmad MS, Mir J, Ullah MO, et al. An efficient heart murmur recognition and cardiovascular disorders classification system. Australas Phys Eng Sci Med. 2019;42:733-743. https://doi.org/10.1007/s13246-019-00778-x

5. Meziani F, Debbal SM, Atbi A. Analysis of phonocardiogram signals using wavelet transform. J Med Eng Technol. 2012;36:283302. https://doi.org/10.3109/03091902.2012.684830

6. Acharya UR, Sudarshan VK, Koh JEW, et al. Application of higher-order spectra for the characterization of Coronary artery disease using electrocardiogram signals. Biomed Signal Process Control. 2017;31:31-43. https://doi.org/10.1016/j.bspc.2016.07.003

7. Mahmoodian N, Schaufler A, Pashazadeh A, et al. Proximal detection of guide wire perforation using feature extraction from bispectral audio signal analysis combined with machine learning. Comput Biol Med. 2019;107:10-17. https://doi.org/10.1016/j.compbiomed.2019.02.001

8. Vejdannik M, Sadr A. Automatic Microstructural Characterization and Classification Using Higher-Order Spectra on Ultrasound Signals. J Nondestruct Eval. 2016;35:16. https://doi.org/10.1007/s10921-015-0332-6

9. Bou Assi E, Gagliano L, Rihana S, et al. Bispectrum Features and Multilayer Perceptron Classifier to Enhance Seizure Prediction. Sci Rep. 2018;8:15491. https://doi.org/10.1038/s41598-018-33969-9

10. Martis RJ, Acharya UR, Adeli H. Current methods in electrocardiogram characterization. Comput Biol Med. 2014;48:133-149. https://doi.org/10.1016/j.compbiomed.2014.02.012

11. Nikias CL, Mendel JM. Signal processing with higher-order spectra. IEEE Signal Process Mag. 1993;10(3):10-37. https://doi.org/10.1109/79.221324

12. Du X, Dua S, Acharya RU, Chua CK. Classification of Epilepsy Using High-Order Spectra Features and Principle Component Analysis. J Med Syst. 2012;36:1731-1743. https://doi.org/10.1007/s10916-010-9633-6

13. Nasrolahzadeh M, Mohammadpoory Z, Haddadnia J. Higher-order spectral analysis of spontaneous speech signals in Alzheimer's disease. Cogn Neurodyn. 2018;12:583-596. https://doi.org/10.1007/s11571-018-9499-8

14. Mishra M, Pratiher S, Banerjee S, Mukherjee A. Grading heart sounds through variational mode decomposition and higher order spectral features. In: 2018 IEEE International Instrumentation and Measurement Technology Conference (I2MTC) 1-5 (IEEE, 2018). https://doi.org/10.1109/I2MTC.2018.8409620

15. Mookiah MRK, Acharya UR, Lim CM, et al. Data mining technique for automated diagnosis of glaucoma using higher order spectra and wavelet energy features. Knowl-Based Syst. 2012;33:73-82. https://doi.org/10.1016/j.knosys.2012.02.010

16. Zhou SM, Gan JQ, Sepulveda F. Classifying mental tasks based on features of higher-order statistics from EEG signals in braincomputer interface. Inf Sci.2008;178:1629-1640. https://doi.org/10.1016/j.ins.2007.11.012

17. Du X, Dua S, Acharya RU, Chua CK. Classification of Epilepsy Using High-Order Spectra Features and Principle Component Analysis. J Med Syst. 2012;36:1731-1743. https://doi.org/10.1007/s10916-010-9633-6

18. Yugesh CK, Hariharan M, Yuvaraj R, et al. Bispectral features and mean shift clustering for stress and emotion recognition from natural speech. Comput Electr Eng. 2017;62:676-691. https://doi.org/10.1016/j.compeleceng.2017.01.024

19. Ahmad TJ, Ali H, Khan SA. Classification of Phonocardiogram using an Adaptive Fuzzy Inference System. Proc. Int. Conf. Image Process. Comput Vis Pattern Recognit. Proceedings of the 2009 International Conference on Image Processing, Computer Vision, \& Pattern Recognition, IPCV 2009, July 13-16, 2009, Las Vegas, Nevada, USA.

20. Meziani F, Debbal SM, Atbi A. Analysis of the pathological severity degree of aortic stenosis (AS) and mitral stenosis (MS) using the discrete wavelet transform (DWT). J Med Eng Technol. 2013;37:61-74. https://doi.org/10.3109/03091902.2012.733058

21. Swami A, Mendel JM, Nikias CL. (1998). Higher-order spectral analysis toolbox. The Mathworks Inc, 3, 22-26.

22. eGeneral Medical Inc. USA. eGeneralMedical.com. http://www.egeneralmedical.com/listohearmur.html Accessed 20 Apr 2018.

23. http://www.cardiosource.com/heart sounds. Accessed 20 Apr 2018. 